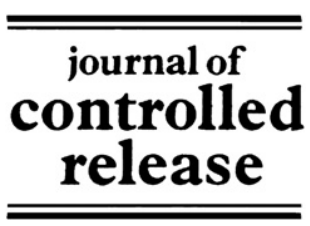

www.elsevier.com/locate/jconrel

\title{
Ultrasound assisted siRNA delivery using PEG-siPlex loaded microbubbles
}

\author{
Roosmarijn E. Vandenbroucke ${ }^{\mathrm{a}, *, 1}$, Ine Lentacker ${ }^{\mathrm{a}, 1}$, Joseph Demeester ${ }^{\mathrm{a}}$, \\ Stefaan C. De Smedt ${ }^{\mathrm{a}, *}$, Niek N. Sanders ${ }^{\mathrm{a}, \mathrm{b}}$ \\ a Laboratory of General Biochemistry and Physical Pharmacy, Ghent University, Harelbekestraat 72, B-9000 Ghent, Belgium \\ ${ }^{\mathrm{b}}$ Laboratory of Veterinary Gene Therapy, Department of Nutrition, Genetics and Ethology, Faculty of Veterinary Medicine, \\ Ghent University, Heidestraat 19, B-9820 Merelbeke, Belgium
}

Received 29 August 2007; accepted 3 December 2007

\begin{abstract}
Short interfering RNA (siRNA) attracts much attention for the treatment of various diseases. However, its delivery, especially via systemic routes, remains a challenge. Indeed, naked siRNAs are rapidly degraded, while complexed siRNAs massively aggregate in the blood or are captured by macrophages. Although this can be circumvented by PEGylation, we found that PEGylation had a strong negative effect on the gene silencing efficiency of siRNA-liposome complexes (siPlexes). Recently, ultrasound combined with microbubbles has been used to deliver naked siRNA but the gene silencing efficiency is rather low and very high amounts of siRNA are required. To overcome the negative effects of PEGylation and to enhance the efficiency of ultrasound assisted siRNA delivery, we coupled PEGylated siPlexes (PEG-siPlexes) to microbubbles. Ultrasound radiation of these microbubbles resulted in massive release of unaltered PEG-siPlexes. Interestingly, PEG-siPlexes loaded on microbubbles were able to enter cells after exposure to ultrasound, in contrast to free PEG-siPlexes, which were not able to enter cells rapidly. Furthermore, these PEG-siPlex loaded microbubbles induced, in the presence of ultrasound, much higher gene silencing than free PEG-siPlexes. Additionally, the PEG-siPlex loaded microbubbles only silenced the expression of genes in the presence of ultrasound, which allows space and time controlled gene silencing.
\end{abstract}

(c) 2007 Elsevier B.V. All rights reserved.

Keywords: siRNA delivery; Delivery vehicle; PEGylation; Ultrasound; Targeting; Microbubbles

\section{Introduction}

RNA interference (RNAi), a naturally occurring process of sequence-specific post-transcriptional gene silencing, is an important biological process for modulating gene expression. The silencing effect of RNAi is highly potent and requires only that the sequence of the target RNA is known. One approach to evoke RNAi in target cells is by the delivery of chemically synthesized siRNAs, which results in a sequence-specific, robust silencing of the targeted gene [1]. The potential of siRNA molecules as therapeutic agent in the treatment of e.g. cancer, viral infections, arthritis, Huntington's disease and

\footnotetext{
* Corresponding authors. Ghent University, Laboratory of General Biochemistry and Physical Pharmacy, Harelbekestraat 72, B-9000 Ghent, Belgium. Tel.: +329 2648047; fax: +3292648189.

E-mail addresses: Roosmarijn.Vandenbroucke@UGent.be

(R.E. Vandenbroucke), Stefaan.DeSmedt@UGent.be (S.C. De Smedt).

${ }^{1}$ Both authors contributed equally to this work.
}

hypercholesterolemia has been widely studied [2]. However, cells do not readily take up siRNAs. Therefore, clinical applications of siRNA largely depend on the development of delivery systems that can bring intact siRNA into the cytoplasm of the target cells of a patient.

Strategies that have been considered for in vivo delivery of synthetic siRNA in laboratory animals are hydrodynamic injection of naked siRNA [3] or siRNA conjugates [4], electroporation [5-8] and the use of cationic carriers [9-14]. However, several aspects limit the applicability of these methods in humans. Indeed, hydrodynamic injection, which involves the intravascular injection of large volumes, generates high pressure in the vascular system and therefore often results in heart failure. Additionally, undesirable gene suppression may be induced in non-target organs. Electroporation allows targeting, but requires the insertion of electrodes into the target area, and hence invasive procedures that limit its range of application. Cationic siRNA delivery carriers, such as cationic lipids and polymers, are often cytotoxic 
and/or not very efficient. Furthermore, they are often not suited for systemic application since their positively charged surface makes them vulnerable to non-specific interactions with blood compounds, leading to life-threatening aggregates and a rapid clearance by the mononuclear phagocyte system $[15,16]$. A common approach for reducing these undesired interactions is by masking the cationic surface of the nanoparticles with hydrophilic polymers, such as polyethylene glycol (PEG). This prevents the aggregation of these nucleic acid containing nanoparticles in blood and prolongs their circulation time [17-20]. However, it has been observed by many groups that shielding the surface of non-viral gene delivery systems with polymers like PEG leads to a drastic reduction in gene transfer, due to a reduced cellular uptake or limited endosomal release $[21,22]$.

The use of ultrasound energy has intensively been studied for pDNA delivery [23-30]. Recently, ultrasound in combination with microbubbles has also been used in two reports to deliver naked siRNA [31,32]. However, the gene silencing efficiency in these studies was rather low and very high amounts of siRNA were required. Nevertheless, ultrasound assisted drug delivery is considered as rather safe as ultrasound, in combination with microbubbles, is routinely used in the clinic for diagnostic purposes. It is believed that ultrasound, especially when combined with microbubbles, causes small (100 to a few $100 \mathrm{~nm}$ large) transient pores in the cell membrane which allows large molecules to enter the cell cytoplasm [33]. These perforations are caused by microjets that are generated by the ultrasound induced cavitation, i.e. alternate growing and shrinking of microbubbles, and implosion of microbubbles. The lifetime of these pores in the cell membranes is very short, i.e. milliseconds to seconds [34], making high concentrations of nucleic acids in the surrounding of the cells beneficial to ensure that a significant amount of nucleic acids can enter the cells through these short-living pores. Consequently, as recently shown by our group for pDNA, microbubbles that at the same time perforate cells and release massive amounts of nucleic acids containing nanoparticles near these perforations may drastically enhance the cellular uptake and hence the biological activity of nucleic acids [35].

Therefore, the aim of this study was to evaluate (a) the loading and release of PEGylated siRNA-liposome complexes (PEG-siPlexes) on/off ultrasound responsive microbubble, and (b) the cellular distribution and gene silencing efficiency of the PEG-siPlex loaded microbubbles after ultrasound radiation.

\section{Materials and methods}

\subsection{Cell culture}

HUH7 and HUH7eGFPLuc cells were cultured in Dulbecco's modified Eagle's medium supplemented with F12 (DMEM: F12) containing $2 \mathrm{mM}$ glutamine, $10 \%$ heat-inactivated FBS and $100 \mathrm{U} / \mathrm{ml}$ penicilline/streptomycine at $37{ }^{\circ} \mathrm{C}$ in a humidified atmosphere containing $5 \% \mathrm{CO}_{2}$. All cell culture products were purchased from Invitrogen (Merelbeke, Belgium).

HUH7eGFPLuc cells stably expressing eGFP-Luciferase were generated by transfecting $\mathrm{HUH} 7$ cells with the vector
pEGFPLuc (Clontech, Palo Alto, USA). The vector was linearized using the restriction enzyme DraIII and transfected using linear polyethylenimine (PEI) $22 \mathrm{kDa}$. Transfected cells were incubated in fresh medium for $72 \mathrm{~h}$ and then selected with 60 to $400 \mu \mathrm{g} / \mathrm{ml} \mathrm{G} 418$. After several days, surviving cells were seeded at low densities into 6-well plates in order to generate separate colonies. Single cell clones were then isolated and expanded. The generated clones were analyzed for the percentage of GFP-positive (eGFPLuc stably transfected) cells. Clones with the highest number of GFP-positive cells were then further selectively grown up under the above described selective conditions and this procedure was repeated until all cells were positive for GFP.

\section{2. $\operatorname{siRNA}$}

Atto488-labelled and non-labelled siRNA duplexes against firefly luciferase and control siRNA duplexes were purchased from Eurogentec (Seraing, Belgium) and dissolved in RNase free water at a final concentration of $20 \mu \mathrm{M}$.

\subsection{Preparation and characterization of lipid microbubbles}

1,2-Dipalmitoyl-sn-glycero-3-phosphocholine (DPPC) and 1,2-distearoyl-sn-glycero-3-phosphoethanolamine- $N$-[biotinyl (polyethylene glycol)2000] (ammonium salt) (DSPE-PEG ${ }_{2000^{-}}$ biotin) were purchased from Avanti Polar Lipids (Alabaster, AL).

Lipid microbubbles were prepared from liposomes composed of DPPC and DSPE-PEG 2000 -biotin with molar ratios of 95:5. Therefore, as described previously [36], appropriate amounts of lipids were dissolved in chloroform and mixed. The chloroform was subsequently removed by rotary evaporation at $37^{\circ} \mathrm{C}$ followed by flushing the obtained lipid film with nitrogen during $30 \mathrm{~min}$ at room temperature. The dried lipids were then hydrated by adding HEPES buffer ( $20 \mathrm{mM}$, pH 7.4) till a final lipid concentration of $5 \mathrm{mg} / \mathrm{ml}$. After mixing in the presence of glass beads, liposome formation was allowed overnight at $4{ }^{\circ} \mathrm{C}$. Thereafter, the DPPC/DSPE-PEG ${ }_{2000}$-biotin liposomes were extruded through two stacked $0.200 \mu \mathrm{m}$ polycarbonate membrane filters (Whatman; Brentfort, UK) at $55{ }^{\circ} \mathrm{C}$ using an Avanti Mini-Extruder (Avanti Polar Lipids). Subsequently, the liposome suspension was sonicated with a $20 \mathrm{kHz}$ probe (Branson 250 sonifier, Branson Ultrasonics Corp.; Danbury, CT) in the presence of perfluorobutane gas (MW 238 g/mol; F2 chemicals; Preston, Lancashire, UK). After sonication, the lipid microbubbles were washed with $3 \mathrm{ml}$ HEPES buffer by $5 \mathrm{~min}$ centrifugation at $470 \mathrm{~g}$. The amount of microbubbles per $\mathrm{ml}$ was determined by light microscopy and equalled $4 \times 10^{8}$ microbubbles $/ \mathrm{ml}$. The size distribution of the microbubbles was determined by laser diffraction (Mastersizer S, Malvern; Worcestershire, UK).

\subsection{Preparation of avidin coated lipid microbubbles}

Avidinylated microbubbles were prepared by incubating them at room temperature with $500 \mu \mathrm{l}$ avidin $(10 \mathrm{mg} / \mathrm{ml})$. After $10 \mathrm{~min}$ of incubation, the microbubbbles were washed with $3 \mathrm{ml}$ HEPES 
buffer by $5 \mathrm{~min}$ centrifugation at $470 \mathrm{~g}$ and finally resuspended in $10 \mathrm{ml}$. For the preparation of red labelled lipid microbubbles, the microbubbles were incubated with the unlabelled avidin supplemented with $50 \mu$ l Cy5-labelled streptavidin $(1 \mathrm{mg} / \mathrm{ml})$ (Zymed Laboratories; San Francisco, CA).

\subsection{Preparation and characterization of liposomes and PEG-siPlexes}

The cationic lipid 1,2-dioleoyl-3-trimethylammonium-propane (chloride salt) (DOTAP) and the helper lipid 1,2-dioleoylsn-glycero-3-phosphoethanolamine (DOPE) were purchased from Avanti Polar Lipids. Cationic liposomes containing DOTAP and DOPE in a 1:1 molar ratio, supplemented with 0 to $15 \mathrm{~mol} \%$ DSPE-PEG 2000 -biotin, were prepared at a final DOTAP concentration of $5 \mathrm{mM}$. All liposomes were prepared as described above for the DPPC/DSPE-PEG ${ }_{2000}$-biotin liposomes, however extrusion occurred through two stacked $0.100 \mu \mathrm{m}$ polycarbonate membrane filters at room temperature. To obtain (PEG-)siPlexes, equal volumes of siRNA solution and extruded liposomes were mixed in a N:P ratio of 20:1. Subsequently, the obtained mixture was vortexed for $5 \mathrm{~s}$ and incubated at room temperature for $30 \mathrm{~min}$.

The average particle size and the zeta potential $(\zeta)$ of the (PEGylated) liposomes and siPlexes were measured by photon correlation spectroscopy (PCS) (Malvern zetasizer nano ZS; Malvern) and by particle electrophoresis (Malvern zetasizer nano ZS; Malvern), respectively. Therefore, the liposome and PEG-siPlex dispersions were diluted 40 -fold in $20 \mathrm{mM}$ HEPES buffer. The size of the liposomes was independent of the degree of PEGylation and averaged $120 \mathrm{~nm}$. In contrast, the zeta potential clearly dropped with increasing degree of PEGylation, varying from $\sim 50 \mathrm{mV}$ for the $0 \mathrm{~mol} \%$ to $\sim 20 \mathrm{mV}$ for the $5 \mathrm{~mol}$ $\%$ DSPE-PEG 2000 -biotin containing DOTAP/DOPE liposomes The size and zeta potential of the siPlexes are displayed in Fig. 5.

\subsection{Preparation and characterization of siPlexes loaded microbubbles}

$130 \mu \mathrm{l}$ siPlex dispersion was mixed with $1 \mathrm{ml}$ microbubbles, vortexed shortly and incubated at room temperature for $5 \mathrm{~min}$. Subsequently, the size distribution of the siPlex loaded microbubbles was determined as described for the non-loaded microbubbles. The time-dependent stability of the (PEG-) siPLex loaded microbubbles was followed for $36 \mathrm{~h}$ at room temperature via light microscopy using a motorized Nikon TE2000-E inverted microscope (Nikon Benelux, Brussels, Belgium). The small microbubbles $(<2 \mu \mathrm{m})$ were stable for at least $24 \mathrm{~h}$ and the larger ones for at least $36 \mathrm{~h}$.

\subsection{Gel electrophoresis}

(PEG-)siPlexes, before binding to the microbubbles and after ultrasound induced release from the microbubbles, were loaded on a native $20 \%$ polyacrylamide gel (PAGE). All samples were supplemented with $10 \%$ glycerol and subjected to electrophoresis at $100 \mathrm{~V}$ for $2 \mathrm{~h}$. Finally, the siRNA was stained with
1:100,000 diluted SYBR-green II dye (Molecular Probes; Merelbeke, Belgium) and visualized by UV transillumination.

\subsection{Confocal laser scanning microscopy}

The Cy5-streptavidin coated microbubbles and Atto488siRNA containing siPlexes were visualized using a Nikon C1si confocal laser scanning module attached to a motorized Nikon TE2000-E inverted microscope (Nikon Benelux; Brussels, Belgium). Images were captured with a $60 \times$ objective lens using the $488 \mathrm{~nm}$ line from an Ar-ion laser for the excitation of Atto488-siRNA and the $639 \mathrm{~nm}$ laser line from a diode laser for the excitation of Cy5-streptavidin.

\subsection{Cellular distribution of PEG-siPlexes in HUH7 cells}

Atto488-siRNA containing PEG-siPlexes (with 5 mol\% DSPE-PEG ${ }_{2000}$-biotin) were prepared as described above. HUH7 cells were grown in OptiCells ${ }^{\mathrm{TM}}$ and incubated for 20 min with the free PEG-siPlexes or with the PEG-siPlexes loaded microbubbles, immediately followed by ultrasound treatment. The ultrasound settings were the same as in the transfection experiments (see below). After one wash step with phosphate buffered saline (PBS; Invitrogen), cells were treated for 10 min with Draq5 (Biostatus Limited; Leicestershire, UK), to stain the nucleus, and TRITC-concanavalin A (Molecular Probes), to stain the cellular membrane. Subsequently, the cellular distribution of the Atto488-siRNA was visualized using a Nikon EZC1-si confocal laser scanning microscope equipped with a $60 \times$ objective. The $488 \mathrm{~nm}$ line of the Ar-ion laser was used to excite the Atto488 label and the $639 \mathrm{~nm}$ line from a diode laser to excite Draq5 and TRITC-concanavalin A.

\subsection{Transfection experiments}

HUH7eGFPLuc cells were seeded in OptiCell ${ }^{\mathrm{TM}}$ units (Biocrystal; Westerville, $\mathrm{OH}$ ) at $4 \times 10^{4}$ cells $/ \mathrm{cm}^{2}$, and allowed to attach overnight in a humidified incubator at $37{ }^{\circ} \mathrm{C}$ and $5 \%$ $\mathrm{CO}_{2}$. The culture medium was removed from the cells and after a washing step with PBS, the free siPlexes or siPlex loaded microbubbles, both dissolved in OptiMEM (Invitrogen) and containing $50 \mathrm{nM}$ siRNA, were added to the OptiCell ${ }^{\mathrm{TM}}$ units. Subsequently, the OptiCell ${ }^{\mathrm{TM}}$ units were placed in a water bath at $37^{\circ} \mathrm{C}$ with an absorbing rubber at the bottom as shown in Fig. 1B and immediately subjected to ultrasound radiation for $10 \mathrm{~s}$ with a sonitron 2000 (RichMar; Inola, OK) equipped with a $22 \mathrm{~mm}$ probe. It has been reported that standing waves can influence the ultrasound assisted transfection efficiency dramatically [37]. In our ultrasound set up (Fig. 1B) standing waves are eliminated as much as possible by (1) using the ultrasound transparent OptiCellTM units, (2) placing an absorbing rubber at the bottom of the water bath and (3) degassing the water. In all experiments the same ultrasound settings were applied: $1 \mathrm{MHz}, 10 \%$ duty cycle (DC) and an ultrasound intensity of $2 \mathrm{~W} / \mathrm{cm}^{2}$ during $10 \mathrm{~s}$. After $2 \mathrm{~h}$ incubation of the cells $\left(\right.$ at $37^{\circ} \mathrm{C}$ ) with the free siPlexes or siPlex loaded microbubbles, the transfection medium was removed, cells were washed twice with PBS and culture medium 


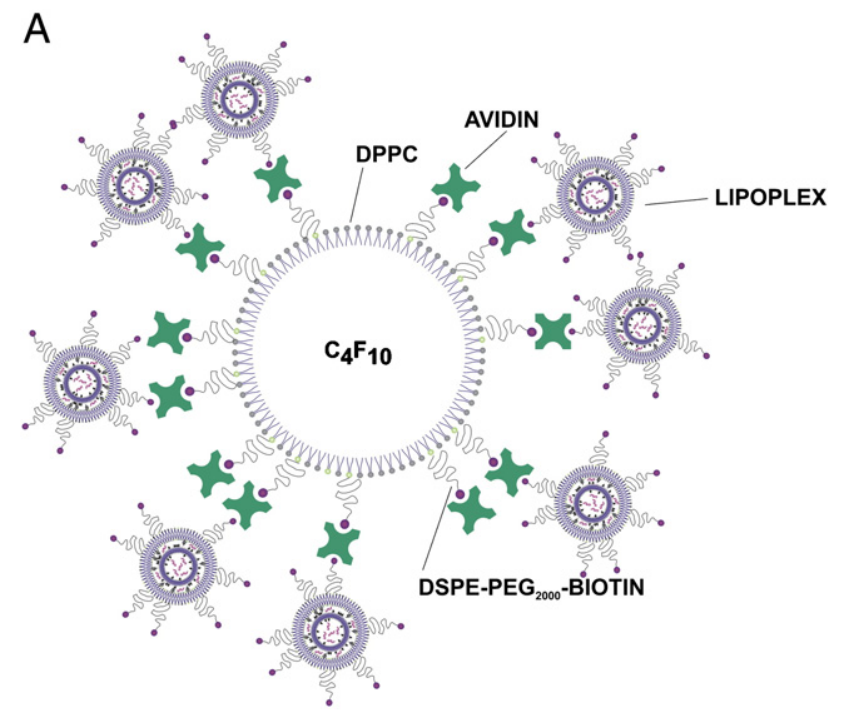

B

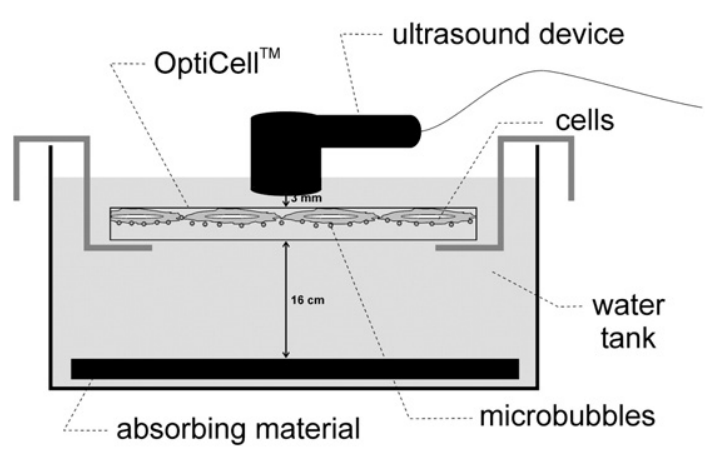

Fig. 1. (A) Schematic overview of a PEG-siPlex loaded microbubble. The white disk surrounded by lipids ( $95 \mathrm{~mol} \%$ DPPC and $5 \mathrm{~mol} \%$ DSPE-PEG-biotin) represents an avidinylated lipid microbubble with its perfluorobutane $\left(\mathrm{C}_{4} \mathrm{~F}_{10}\right)$ gas core. PEG-siPlexes with increasing amounts of DSPE-PEG-biotin were attached to these avidinylated microbubbles via a biotin-avidin-biotin bridge. (B) Experimental setup used in the transfection experiments. An OptiCell ${ }^{\mathrm{TM}}$ unit containing a monolayer of $\mathrm{HUH7}$ cells on one of their membranes was placed in a water tank with a rubber plate, designed to minimize ultrasound reflection or scattering, at the bottom. In all experiments the same ultrasound settings were applied: $10 \mathrm{~s}, 1 \mathrm{MHz}, 10 \%$ duty cycle and an ultrasound intensity of $2 \mathrm{~W} / \mathrm{cm}^{2}$. The ultrasound was delivered vertically to the cells which were present on the upper membrane of the OptiCell ${ }^{\mathrm{TM}}$ unit, closest to the ultrasound probe. Different regions of the OptiCell ${ }^{\mathrm{TM}}$ unit were sonicated separately by moving the ultrasound device.

was added. After $48 \mathrm{~h}$ incubation, discs $(22 \mathrm{~mm}$ in diameter) were cut from the OptiCell ${ }^{\mathrm{TM}}$ membrane, transferred to a 24-well plate and lysed with $80 \mu 11 \times$ CCLR buffer (Promega; Leiden, The Netherlands) to measure both the luciferase activity and the total protein concentration.

Luciferase activity was determined with the Promega luciferase assay kit according to the manufacturer's instructions in relative light units (RLU). Briefly, $100 \mu 1$ substrate was added to $20 \mu \mathrm{l}$ cell lysate and after a $2 \mathrm{~s}$ delay, the luminescence was measured during $10 \mathrm{~s}$ with a GloMax ${ }^{\mathrm{TM}} 96$ luminometer. To correct for the amount of cells per well, the protein concentration was determined with the BCA kit (Pierce; Rockford, IL). Therefore, $200 \mu 1$ mastermix, containing 50 parts reagent $A$ to 1 part B, was mixed with $20 \mu \mathrm{l}$ cell lysate or BSA (to make the standard curve). After 30 min incubation at $37{ }^{\circ} \mathrm{C}$, the absorbance at $590 \mathrm{~nm}$ was measured with a Wallac Victor2 absorbance plate reader (Perkin Elmer; Waltham, MA).

\section{Results and discussion}

\subsection{Preparation and characterization of PEG-siPlex loaded microbubbles}

As schematically depicted in Fig. 1A, the first goal of this work was to attach PEGylated siRNA-liposome complexes (PEGsiPlexes) to gas-filled microbubbles via a biotin-avidin-biotin bridge. Therefore, we first prepared perfluorobutane filled lipid microbubbles by sonication of a DPPC/DSPE- EEG $_{2000}$-biotin liposome dispersion in the presence of perfluorobutane gas. The lipid coating prevents a rapid diffusion of the perfluorobutane gas out of the microbubbles. To assure that biotin molecules were present at the outer surface of the microbubbles, we incubated them with Cy5-labelled streptavidin. As shown by the confocal images in Fig. 2A and B, after removal of the unbound streptavidin, a thin fluorescent layer of streptavidin molecules surrounding the gas-filled microbubbles could be observed, which indicates the formation of biotin-avidin linkages. This suggests
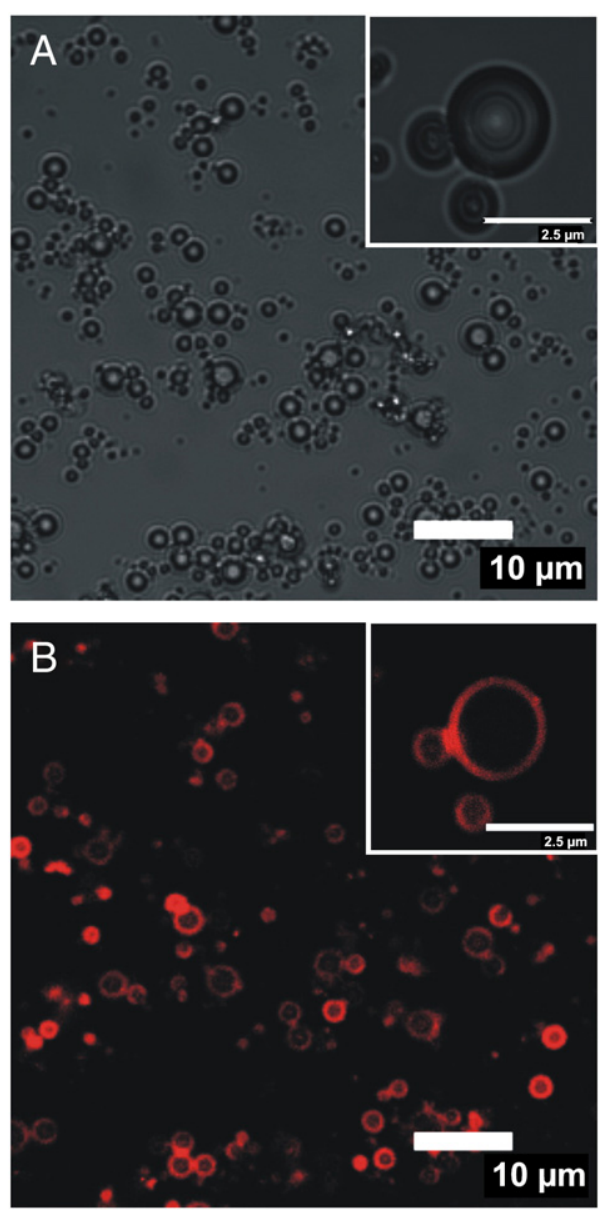

Fig. 2. Transmission image (A) and confocal laser scanning microscopy image (B) of avidinylated microbubbles coated with Cy5-streptavidin (red). The inserts display a close-up of three microbubbles. 
that the DSPE-PEG-biotin molecules in the lipid shell are oriented with their hydrophobic tails to the perfluorobutane gas core while their hydrophilic head groups are exposed to the surrounding aqueous medium, as previously suggested by Unger [38].

In a next step, we prepared DOTAP/DOPE based siPlexes containing increasing amounts of DSPE-PEG 2000 -biotin $(0,2,5$ and $15 \mathrm{~mol} \%$ ). In all cases, a N:P ratio of 20:1 was chosen as non-PEGylated siPlexes showed at this ratio the highest gene silencing effect in HUH7 cells (data not shown). The ability of the siPlexes to bind siRNA was analyzed by PAGE (Fig. 3A). In

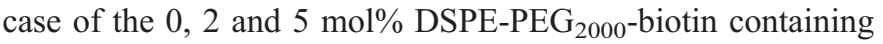
siPlexes, no free siRNA could be detected. This implies that all the siRNA is complexed with the liposomes, which are too large to migrate into the gel network. In contrast, a smear of siRNA was observed in case of the $15 \mathrm{~mol} \%$ PEG-siPlexes, indicating only a partial siRNA complexation in these siPlexes.

Subsequently, the ability of the different siPlexes to bind to the surface of the biotinylated microbubbles was tested. Therefore, the biotinylated microbubbles were first incubated with avidin. An excess of avidin was used to avoid massive clustering of the microbubbles, due to avidin mediated bridging.
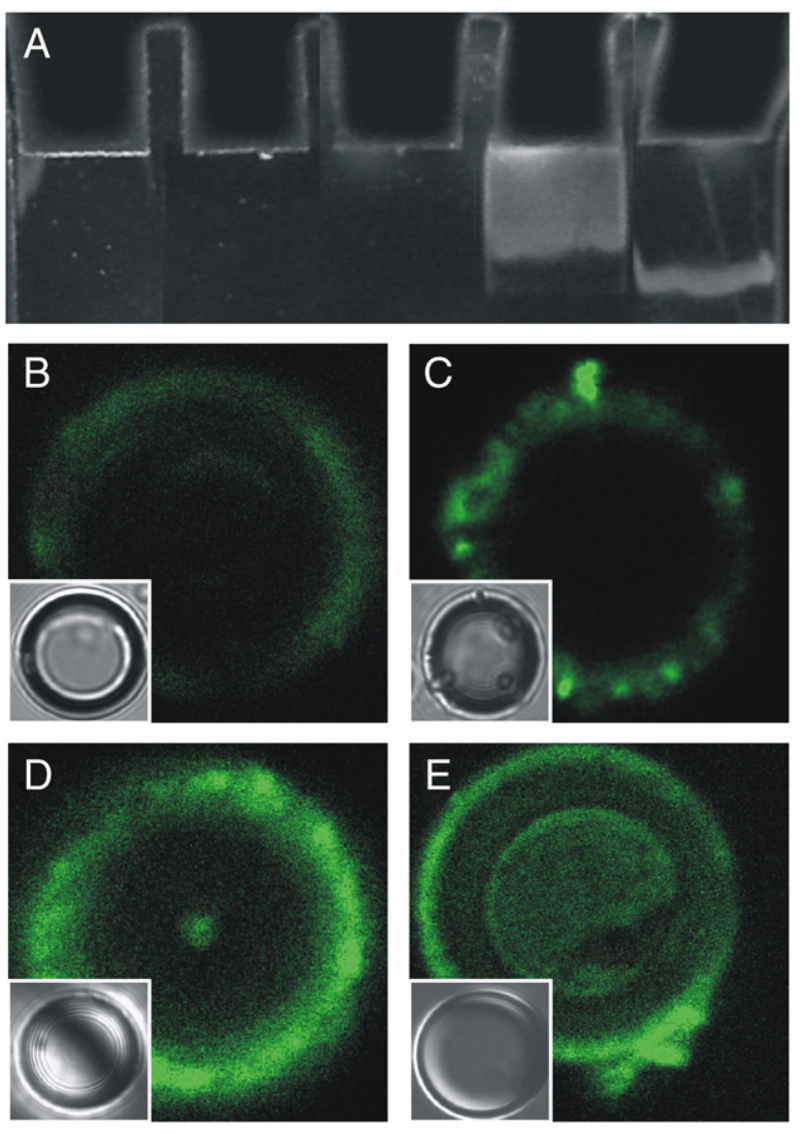

Fig. 3. (A) Polyacrylamide gel after electrophoresis of siPlexes containing $0 \mathrm{~mol}$ $\%$ (lane 1), 2 mol\% (lane 2), 5 mol\% (lane 3) and 15 mol\% DSPE-PEG $2000^{-}$ biotin (lane 4) before attachment to the microbubbles. As a reference, free siRNA was loaded in lane 5 and each lane contains $0.3 \mu \mathrm{g}$ siRNA. (B-E) Confocal laser scanning microscopy images and corresponding transmission images (inserts) of avidinylated microbubbles incubated with siPlexes containing $0 \mathrm{~mol} \%$ (B), $2 \mathrm{~mol} \%$ (C), $5 \mathrm{~mol} \%$ (D) and $15 \mathrm{~mol} \%$ DSPE $\mathrm{PEG}_{2000}$-biotin (E). The siPlexes were visualized by using Atto488-siRNA.
After removal of the unbound avidin, siPlexes, containing Atto488 labelled siRNA, were added to the avidinylated microbubbles. The confocal images in Fig. 3B till E show that the amount of DSPE-PEG ${ }_{2000}$-biotin in the siPlexes clearly influences to which extent the microbubble surface becomes covered with siPlexes. Non-PEGylated siPlexes (Fig. 3B), thus not containing DSPE-PEG ${ }_{2000}$-biotin, only showed some nonspecific binding to the avidinylated microbubbles. In contrast, the PEGylated siPlexes, containing DSPE-PEG ${ }_{2000}$-biotin, clearly bound to the avidinylated surface of the microbubbles. The surface of the microbubbles became only partially covered with siPlexes containing $2 \mathrm{~mol} \%$ DSPE-PEG 2000 -biotin (Fig. 3C), probably due to the limited degree of biotinylation of the siPlexes. In contrast, the microbubble surface was almost completely coated with siPlexes when they contained 5 mol\% DSPE$\mathrm{PEG}_{2000}$-biotin (Fig. 3D). The $15 \mathrm{~mol} \%$ containing PEG-siPlexes also showed an efficient coating of the surface of the microbubbles. However, as these siPlexes showed incomplete complexation of the siRNA, previously shown by gel electrophoresis experiments (Fig. 3A, lane 5), these siPlexes were further excluded from the study.

Subsequently we studied the size distribution of the siPlex coated microbubbles by laser diffraction to assure that the microbubbles had the optimal size distribution for cavitation. Fig. 4 shows the size distribution of microbubbles incubated with non-PEGylated siPlexes and microbubbles loaded with PEGylated siPlexes. In both cases, the diameter of the microbubbles varied between 0.5 and $10 \mu \mathrm{m}$, which is an appropriate size to favour cavitation upon exposure to clinically relevant ultrasound energy [38]. Fig. 4 (arrow) also shows a significant amount of sub-micron particles in case of the non-PEGylated siPlexes, indicating the presence of non-bound siPlexes. In contrast, this sub-micron peak was not visible in case of the microbubbles loaded with PEGylated siPlexes (Fig. 4, grey circles). These results are in agreement with the confocal images shown in Fig. 3B and D. Similar size distributions were found for microbubbles that were loaded with $2 \mathrm{~mol} \%$ DSPE$\mathrm{PEG}_{2000}$-biotin containing siPlexes (data not shown).

\subsection{Ultrasound induced release of PEG-siPlexes from microbubbles}

It has been shown that coupling of polystyrene beads to the surface of microbubbles via a biotin-avidin bridge, results in local delivery of the beads upon ultrasound radiation [39]. However, in contrast to these inert beads, self-assembled siPlexes may undergo physicochemical alterations during the ultrasound triggered release, which may influence their biological performance. Therefore, we determined the size, zeta potential and siRNA complexation of the siPlexes before attachment to the microbubbles and after ultrasound triggered release from the microbubbles.

The dark grey bars in Fig. 5A show that, before binding to the microbubble surface the size of the siPlexes was independent of the PEGylation degree and averaged $130 \mathrm{~nm}$. In contrast, the surface charge lowered with increasing degree of PEGylation (dark grey bars in Fig. 5B) varying from $50 \mathrm{mV}$ for the non- 


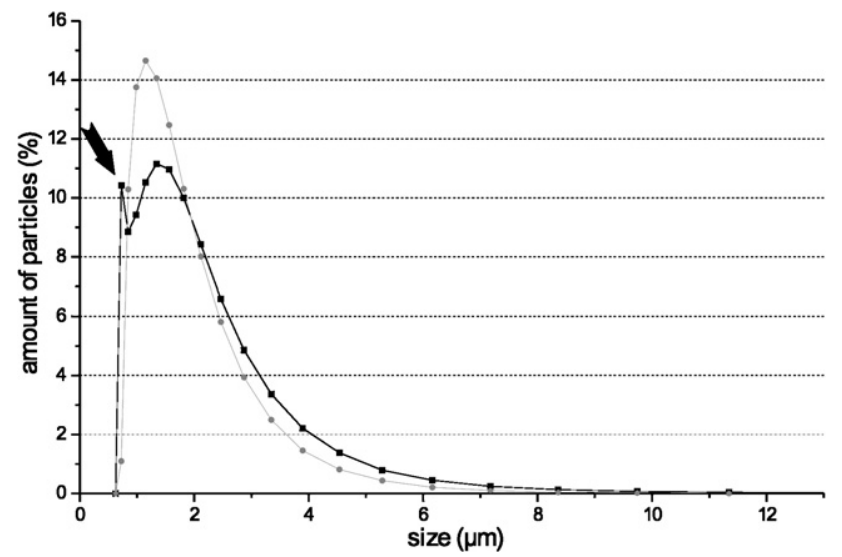

Fig. 4. Size distribution measured by laser diffraction of microbubbles after addition of non-PEGylated siPlexes (black squares) and attachment of $5 \mathrm{~mol} \%$ DSPE-PEG $_{2000}$-biotin containing siPlexes (grey circles). The data are the mean of three measurements and error bars represent standard deviations. Arrow indicates a peak of sub-micron sized particles.

PEGylated to $20 \mathrm{mV}$ for the siPlexes containing $5 \mathrm{~mol} \%$ DSPE$\mathrm{PEG}_{2000}$-biotin. Fig. 5A also shows that binding and subsequent ultrasound assisted release of the PEG-siPlexes from the microbubbles (light grey bars) had only a limited effect on the size of these PEG-siPlexes with a maximal increase of $\sim 20 \mathrm{~nm}$, in contrast to the clear increase in size of the non-PEGylated siPlexes.

As observed for the size, the zeta potential (Fig. 5B) of the PEG-siPlexes after being released from the microbubbles by ultrasound was not significantly altered, while the zeta potential of the non-PEGylated siPlexes was significantly lower. The change in size and zeta potential of the non-PEGylated siPlexes may be due the binding of negatively charged DSPE-PEG ${ }_{2000^{-}}$ biotin lipids from the imploded microbubbles to the nonPEGylated siPlexes.

Clearly, to keep their biological performance, the siPlexes may not dissociate (i.e. release their siRNA) upon exposure to ultrasound, as free siRNA is prone to nuclease degradation. Gel electrophoresis revealed that ultrasound energy did not dissociate the siPlexes (data not shown). In conclusion, ultrasoundmediated implosion of the PEG-siPlex loaded microbubbles and the induced microjets did not drastically influence the size, zeta potential and the complexation properties of the released PEGsiPlexes.

\subsection{Cellular distribution of PEG-siPlexes}

Next we studied the cellular distribution of PEG-siPlexes. Fig. 6A till C shows $\mathrm{HUH} 7$ cells incubation with $5 \mathrm{~mol} \%$ PEGsiPlexes. The $z$-scan in Fig. 6A reveals that these PEG-siPlexes, after 20 min incubation at $37^{\circ} \mathrm{C}$, were still located on top of the HUH7 cells. This was confirmed by the images in Fig. 6B and C. In these images, the green labelled PEG-siPlexes (Fig. 6C) show exactly the same cellular distribution as the red labelled plasma membrane (Fig. 6B). This confirms that PEGylation indeed has an effect on the cellular uptake of siPlexes [40], as non-PEGylated siPlexes were clearly taken up by the cells after 20 min (data not shown). Ultrasound irradiation did not change the cellular distribution of these free PEG-siPlexes (data not shown). Interestingly, PEG-siPlexes released from siPlex loaded microbubbles by ultrasound showed a totally different cellular distribution (Fig. 6D till F). In this case, the green labelled PEG-siPlexes were localized inside the cells as shown by the $z$-scan (Fig. 6D) and the membrane colouring (Fig. 6E and F). These results suggest that PEG-siPlexes enter the cells via a different mechanism when they are released from the PEGsiPLex loaded microbubbles by ultrasound. Although further research is needed, we suppose that they enter cells via the transient cell membrane perforations that arise during the exposure to ultrasound [41]. Indeed, such pores, which have been reported to be a few hundreds of nanometers in size $[34,42]$, are large enough to allow the passage of the PEGsiPlexes released from the microbubbles. This implies that the negative effects of PEGylation on the cellular uptake as well as on the endosomal escape of PEG-siPlexes can be circumvented by attaching them to microbubbles and subsequently expose these siPlex loaded microbubbles to ultrasound.

\subsection{Gene silencing efficiency of PEG-siPlex loaded micro- bubbles}

Finally, we determined whether siRNA delivered by ultrasound-mediated implosion of the PEG-siPLex loaded microbubbles could inhibit constitutive luciferase expression in

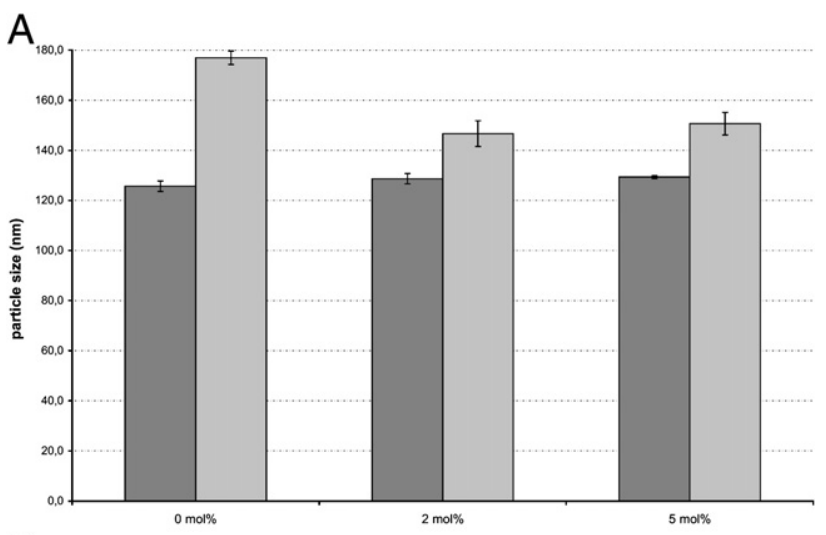

B

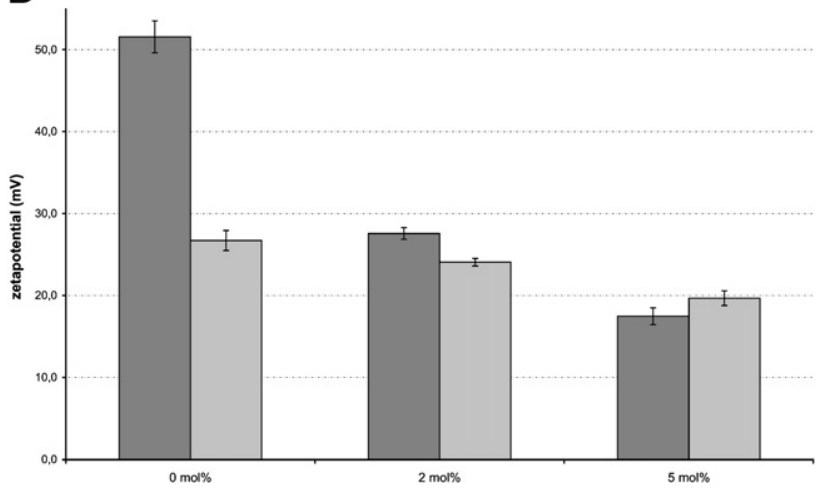

Fig. 5. (A) Size and (B) zeta potential of the siPlexes containing $0 \mathrm{~mol} \%, 2 \mathrm{~mol} \%$ and $5 \mathrm{~mol} \%$ DSPE-PEG 2000 -biotin before attachment to the microbubbles (dark grey bars) and after ultrasound assisted release from the siPlex loaded microbubbles (light grey bars). The data are the means of three independent measurements and the error bars represent standard deviations. 

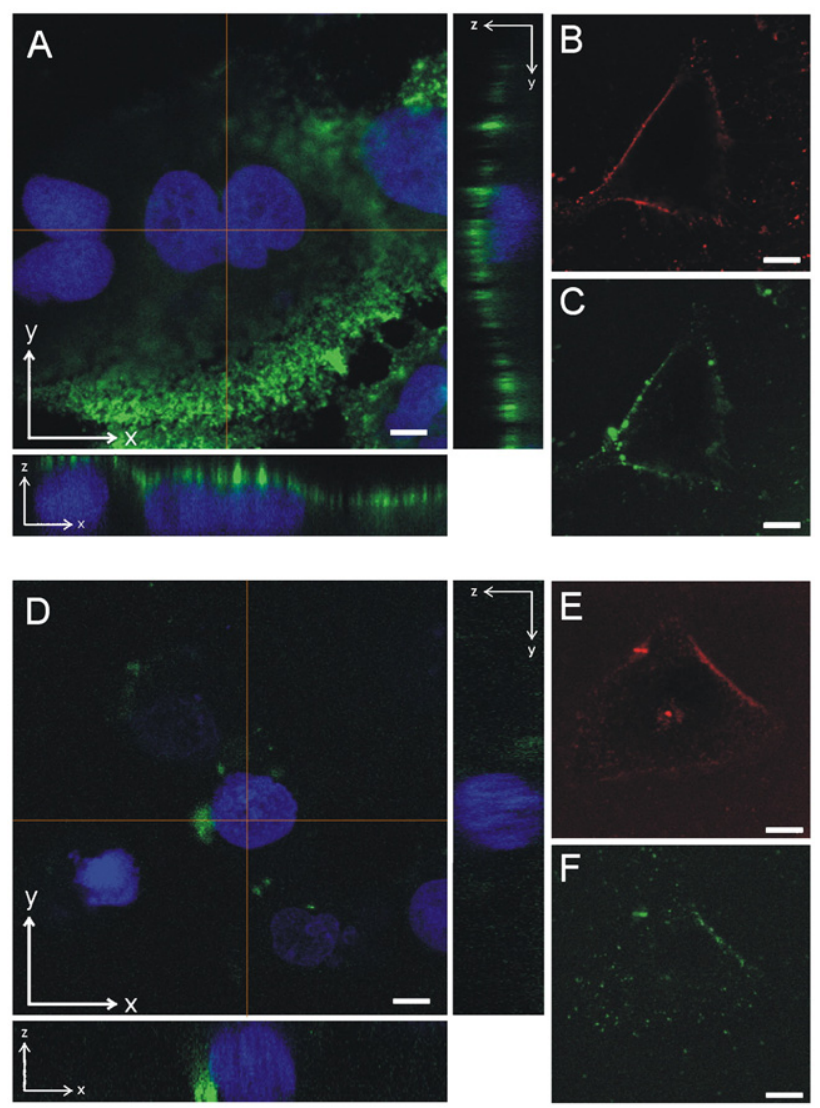

Fig. 6. Cellular uptake and intracellular distribution of PEG-siPlexes (A till C) and ultrasound irradiated PEG-siPlex loaded microbubbles (D till F). All PEGsiPlexes contain $5 \mathrm{~mol} \%$ DSPE-PEG ${ }_{2000}$-biotin. Images $\mathrm{A}$ and D display confocal images and $z$-scans, at the positions indicated by the red lines, through HUH7 cells with Draq5 labelled nuclei (blue) and incubated for 20 min with Atto488-labelled siPlexes (green). Confocal images (B) and (E) show the localisation of TRITC-concanavalin A (red), a plasma membrane marker. Confocal images (C) and (F) show the localisation of Atto488-labelled siPlexes (green) in the cells shown in images (B) and (E), respectively. The scale bars represent $10 \mu \mathrm{m}$.

HUH7eGFPLuc cells (Fig. 7). The black bars in Fig. 7 show that the silencing capacity of the free siPlexes declines dramatically with increasing PEGylation degree. siPlexes with a PEGylation degree of $2 \mathrm{~mol} \%$ already showed a 3 -fold reduced gene silencing capacity compared to the non-PEGylated siPlexes. Increasing the PEGylation degree to $5 \mathrm{~mol} \%$ even completely blocked the silencing capacity of the siPlexes. This negative effect of PEGylation has intensively been studied for pDNA delivery and some groups suggest that the loss in transfection efficiency of highly PEGylated lipoplexes is due to a reduced cellular binding and uptake [43], while others believe that the PEG-lipids inhibit the endosomal release of the nucleic acids into the cytoplasm [4448]. The white bars in Fig. 7 show that the negative effect of PEGylation on the gene silencing efficiency of the siPlexes containing $5 \mathrm{~mol} \%$ DSPE-PEG 2000 -biotin can be completely counteracted by loading of these PEG-siPlexes on the surface of microbubbles followed by exposure of these microbubbles to ultrasound. Attachment of siPlexes containing 2 mol\% DSPE$\mathrm{PEG}_{2000}$-biotin to the microbubbles and subsequent exposure to ultrasound resulted in a similar silencing as the corresponding free PEG-siPlexes. For these PEG-siPlexes the number of PEGsiPlexes bound to the microbubbles is probably not enough to further increase their gene silencing efficiency. Indeed, as shown in Fig. $3 \mathrm{C}$, the $5 \mathrm{~mol} \%$ DSPE-PEG 2000 -biotin containing siPlexes bind much more efficiently to the microbubble surface than the 2 mol\% DSPE-PEG 2000 -biotin containing siPlexes (Fig. 3B). Therefore, these data may indicate that the extent of gene silencing is governed by the amount of PEG-siPlexes that are released near the cell perforations. Fig. 7 (dark grey bars) also shows that applying ultrasound energy, in the absence of microbubbles, could not enhance the gene silencing efficiency of the different siPlexes. Furthermore, microbubbles loaded with 5 mol\% PEG-siPlexes were not able to cause gene silencing in the absence of ultrasound (Fig. 7; light grey bars). This implies that the PEG-siPlex loaded microbubbles described in this work allow ultrasound controlled, i.e. targeted, intracellular delivery of siRNA.

\section{Conclusion}

In conclusion, we developed a novel delivery system in which PEG-siPlexes are attached to ultrasound responsive microbubbles via a biotin-avidin-biotin bridge. Exposure of these PEG-siPlex loaded microbubbles to ultrasound resulted in a massive release of unaltered PEG-siPlexes. Furthermore, PEG-siPlexes (containing $5 \mathrm{~mol} \%$ DSPE-PEG 2000 -biotin) loaded on microbubbles were able to enter cells rapidly after exposure to ultrasound, while free PEG-siPlexes did not enter cells. Moreover, these PEG-siPlex loaded microbubbles caused,

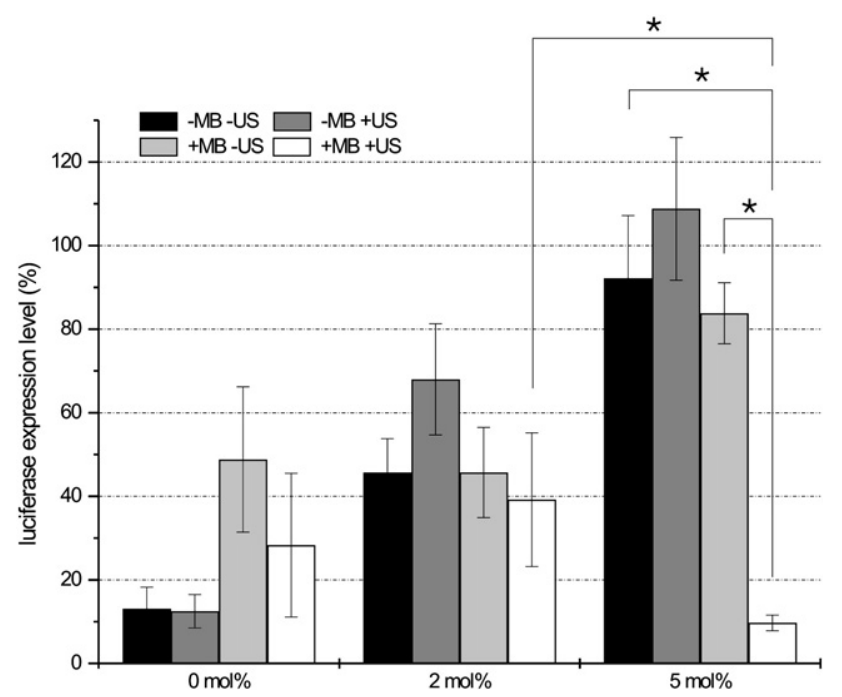

Fig. 7. Silencing efficiency of free siPlexes and PEG-siPlexes in the absence and presence of ultrasound, and PEG-siPlex loaded microbubbles in the presence of ultrasound. The PEGylation (DSPE-PEG 2000 -biotin) degree of the siPlexes is represented in the $x$-axis. The black and the dark grey bars represent the gene silencing efficiency of free siPlexes in the absence and presence of ultrasound, respectively. The light grey and white bars represent the gene silencing of the siPlexes loaded on the microbubbles in the absence or presence of ultrasound. In all cases, the following ultrasound settings were used: $1 \mathrm{MHz}, 10 \% \mathrm{DC}, 2 \mathrm{~W} / \mathrm{cm}^{2}$, $10 \mathrm{~s}$. The results are expressed as percentage of luciferase expression compared to mock siRNA transfected cells. The asterisks $(*)$ represent significant differences with $p<0.05$. ( $\mathrm{MB}=$ microbubble; $\mathrm{US}=$ ultrasound $)$. 
in the presence of ultrasound, a much higher gene silencing than free PEG-siPlexes. Interestingly, in the absence of ultrasound these PEG-siPlex loaded microbubbles did not cause any gene silencing. Therefore, the developed siRNA delivery system allows both space and time controlled gene silencing. Furthermore, the PEG-siPlex loaded microbubbles are expected to be suitable for systematic applications as ultrasound in combination with microbubbles is considered as a safe and already used in the clinic for diagnostic purposes. Additionally, PEGsiPlexes are known not to aggregate in serum which is important to avoid blockage of small blood capillaries by aggregates [49]. The developed siRNA delivery system may also allow the treatment of patients with metastasized tumours. Indeed, a recently developed device that combines magnetic resonance imaging (MRI) and ultrasound can both track down the metastasized tumours and guide the ultrasound energy to these tumours [50]. So, the siRNA delivery system presented in this work may open up new perspectives for ultrasound controlled in vivo delivery of siRNA.

\section{Acknowledgements}

Niek Sanders is supported by the Fund for Scientific Research-Flanders (FWO). The financial support of this institute is acknowledged with gratitude. This work was supported by grants from Ghent University (BOF), FWO and the European Union (MediTrans). The HUH7eGFPLuc cells were kindly provided by Ernst Wagner, LMU University Munich.

\section{References}

[1] S.M. Elbashir, J. Harborth, W. Lendeckel, A. Yalcin, K. Weber, T. Tuschl, Duplexes of 21-nucleotide RNAs mediate RNA interference in cultured mammalian cells, Nature 411 (6836) (2001) 494-498.

[2] L. Aagaard, J.J. Rossi, RNAi therapeutics: principles, prospects and challenges, Adv. Drug Deliv. Rev. 59 (2-3) (2007) 75-86.

[3] D.L. Lewis, J.A. Wolff, Systemic siRNA delivery via hydrodynamic intravascular injection, Adv. Drug Deliv. Rev. 59 (2-3) (2007) 115-123.

[4] S.H. Lee, S.H. Kim, T.G. Park, Intracellular siRNA delivery system using polyelectrolyte complex micelles prepared from VEGF siRNA-PEG conjugate and cationic fusogenic peptide, Biochem. Biophys. Res. Commun. 357 (2) (2007) 511-516.

[5] Y. Akaneya, B. Jiang, T. Tsumoto, RNAi-induced gene silencing by local electroporation in targeting brain region, J. Neurophysiol. 93 (1) (2005) 594-602.

[6] M. Golzio, L. Mazzolini, A. Ledoux, et al., In vivo gene silencing in solid tumors by targeted electrically mediated siRNA delivery, Gene Ther. 14 (9) (2007) 752-759.

[7] T. Kishida, H. Asada, S. Gojo, et al., Sequence-specific gene silencing in murine muscle induced by electroporation-mediated transfer of short interfering RNA, J. Gene Med. 6 (1) (2004) 105-110.

[8] Y. Takabatake, Y. Isaka, M. Mizui, et al., Exploring RNA interference as a therapeutic strategy for renal disease, Gene Ther. 12 (12) (2005) 965-973.

[9] P.Y. Chien, J. Wang, D. Carbonaro, et al., Novel cationic cardiolipin analogue-based liposome for efficient DNA and small interfering RNA delivery in vitro and in vivo, Cancer Gene Ther. 12 (3) (2005) 321-328.

[10] K.A. Howard, U.L. Rahbek, X. Liu, et al., RNA interference in vitro and in vivo using a novel chitosan/siRNA nanoparticle system, Mol. Ther. 14 (4) (2006) 476-484.

[11] R.M. Schiffelers, A. Ansari, J. Xu, et al., Cancer siRNA therapy by tumor selective delivery with ligand-targeted sterically stabilized nanoparticle, Nucleic Acids Res. 32 (19) (2004) e149.
[12] D.R. Sorensen, M. Leirdal, M. Sioud, Gene silencing by systemic delivery of synthetic siRNAs in adult mice, J. Mol. Biol. 327 (4) (2003) 761-766.

[13] M. Thomas, J.J. Lu, Q. Ge, C. Zhang, J. Chen, A.M. Klibanov, Full deacylation of polyethylenimine dramatically boosts its gene delivery efficiency and specificity to mouse lung, Proc. Natl. Acad. Sci. U. S. A. 102 (16) (2005) 5679-5684.

[14] B. Urban-Klein, S. Werth, S. Abuharbeid, F. Czubayko, A. Aigner, RNAimediated gene-targeting through systemic application of polyethylenimine (PEI)-complexed siRNA in vivo, Gene Ther. 12 (5) (2005) 461-466.

[15] H. Eliyahu, N. Servel, A.J. Domb, Y. Barenholz, Lipoplex-induced hemagglutination: potential involvement in intravenous gene delivery, Gene Ther. 9 (13) (2002) 850-858.

[16] F. Sakurai, T. Nishioka, F. Yamashita, Y. Takakura, M. Hashida, Effects of erythrocytes and serum proteins on lung accumulation of lipoplexes containing cholesterol or DOPE as a helper lipid in the single-pass rat lung perfusion system, Eur. J. Pharm. Biopharm. 52 (2) (2001) 165-172.

[17] M. Ogris, S. Brunner, S. Schuller, R. Kircheis, E. Wagner, PEGylated DNA/transferrin-PEI complexes: reduced interaction with blood components, extended circulation in blood and potential for systemic gene delivery, Gene Ther. 6 (4) (1999) 595-605.

[18] Y.H. Choi, F. Liu, J.S. Kim, Y.K. Choi, J.S. Park, S.W. Kim, Polyethylene glycol-grafted poly-L-lysine as polymeric gene carrier, J. Control. Release 54 (1) (1998) 39-48

[19] K. Kunath, A. von Harpe, H. Petersen, et al., The structure of PEGmodified poly(ethylene imines) influences biodistribution and pharmacokinetics of their complexes with NF-kappaB decoy in mice, Pharm. Res. 19 (6) (2002) 810-817.

[20] P. Tam, M. Monck, D. Lee, et al., Stabilized plasmid-lipid particles for systemic gene therapy, Gene Ther. 7 (21) (2000) 1867-1874.

[21] L. Wasungu, D. Hoekstra, Cationic lipids, lipoplexes and intracellular delivery of genes, J. Control. Release 116 (2) (2006) 255-264.

[22] I.S. Zuhorn, J.B. Engberts, D. Hoekstra, Gene delivery by cationic lipid vectors: overcoming cellular barriers, Eur. Biophys. J. 36 (4-5) (2006) 349-362.

[23] R. Bekeredjian, S. Chen, P.A. Frenkel, P.A. Grayburn, R.V. Shohet, Ultrasound-targeted microbubble destruction can repeatedly direct highly specific plasmid expression to the heart, Circulation 108 (8) (2003) $1022-1026$.

[24] M. Duvshani-Eshet, D. Adam, M. Machluf, The effects of albumin-coated microbubbles in DNA delivery mediated by therapeutic ultrasound, J. Control. Release 112 (2) (2006) 156-166.

[25] M. Kinoshita, N. McDannold, F.A. Jolesz, K. Hynynen, Targeted delivery of antibodies through the blood-brain barrier by MRI-guided focused ultrasound, Biochem. Biophys. Res. Commun. 340 (4) (2006) 1085-1090.

[26] I. Lentacker, S.C. De Smedt, J. Demeester, V. Van Marck, M. Bracke, N.N Sanders, Lipoplex-loaded microbubbles for gene delivery: a Trojan horse controlled by ultrasound, Adv. Funct. Mater. 17 (12) (2007) 1910-1916.

[27] Y. Manome, N. Nakayama, K. Nakayama, H. Furuhata, Insonation facilitates plasmid DNA transfection into the central nervous system and microbubbles enhance the effect, Ultrasound Med. Biol. 31 (5) (2005) 693-702.

[28] C.M. Newman, A. Lawrie, A.F. Brisken, D.C. Cumberland, Ultrasound gene therapy: on the road from concept to reality, Echocardiography 18 (4) (2001) 339-347.

[29] S.V. Pislaru, C. Pislaru, R.R. Kinnick, et al., Optimization of ultrasoundmediated gene transfer: comparison of contrast agents and ultrasound modalities, Eur. Heart J. 24 (18) (2003) 1690-1698.

[30] M. Vannan, T. McCreery, P. Li, et al., Ultrasound-mediated transfection of canine myocardium by intravenous administration of cationic microbubblelinked plasmid DNA, J. Am. Soc. Echocardiogr. 15 (3) (2002) 214-218.

[31] M. Kinoshita, K. Hynynen, A novel method for the intracellular delivery of siRNA using microbubble-enhanced focused ultrasound, Biochem. Biophys. Res. Commun. 335 (2) (2005) 393-399.

[32] S. Tsunoda, O. Mazda, Y. Oda, et al., Sonoporation using microbubble BR14 promotes pDNA/siRNA transduction to murine heart, Biochem. Biophys. Res. Commun. 336 (1) (2005) 118-127.

[33] K. Ogawa, K. Tachibana, T. Uchida, et al., High-resolution scanning electron microscopic evaluation of cell-membrane porosity by ultrasound, Med. Electron Microsc. 34 (4) (2001) 249-253. 
[34] S. Mehier-Humbert, T. Bettinger, F. Yan, R.H. Guy, Ultrasound-mediated gene delivery: kinetics of plasmid internalization and gene expression, J. Control. Release 104 (1) (2005) 203-211.

[35] I. Lentacker, S.C. De Smedt, J. Demeester, V. Van Marck, M. Bracke, N.N. Sanders, Lipoplex-loaded microbubbles for gene delivery: a Trojan horse controlled by ultrasound, Adv. Funct. Mater. 17 (12) (2007) 1910-1916.

[36] N.N. Sanders, R.E. Van, S.C. De Smedt, J. Demeester, Structural alterations of gene complexes by cystic fibrosis sputum, Am. J. Respir. Crit Care Med. 164 (3) (2001) 486-493.

[37] M. Kinoshita, K. Hynynen, Key factors that affect sonoporation efficiency in in vitro settings: the importance of standing wave in sonoporation, Biochem. Biophys. Res. Commun. 359 (4) (2007) 860-865.

[38] E.C. Unger, T. Porter, W. Culp, R. Labell, T. Matsunaga, R. Zutshi, Therapeutic applications of lipid-coated microbubbles, Adv. Drug Deliv. Rev. 56 (9) (2004) 1291-1314.

[39] A.F. Lum, M.A. Borden, P.A. Dayton, D.E. Kruse, S.I. Simon, K.W. Ferrara, Ultrasound radiation force enables targeted deposition of model drug carriers loaded on microbubbles, J. Control. Release 111 (1-2) (2006) 128-134.

[40] S. Mishra, P. Webster, M.E. Davis, PEGylation significantly affects cellular uptake and intracellular trafficking of non-viral gene delivery particles, Eur. J. Cell Biol. 83 (3) (2004) 97-111.

[41] P. Prentice, A. Cuschieri, K. Dholakia, M. Prausnitz, P. Campbell, Membrane disruption by optically controlled microbubble cavitation, Nature Physics 1 (2005) 107-110.

[42] R.K. Schlicher, H. Radhakrishna, T.P. Tolentino, R.P. Apkarian, V. Zarnitsyn, M.R. Prausnitz, Mechanism of intracellular delivery by acoustic cavitation, Ultrasound Med. Biol. 32 (6) (2006) 915-924.
[43] M.C. Deshpande, M.C. Davies, M.C. Garnett, et al., The effect of poly (ethylene glycol) molecular architecture on cellular interaction and uptake of DNA complexes, J. Control. Release 97 (1) (2004) 143-156.

[44] S. Audouy, D. Hoekstra, Cationic lipid-mediated transfection in vitro and in vivo, Mol. Membr. Biol. 18 (2) (2001) 129-143.

[45] O. Meyer, D. Kirpotin, K.L. Hong, et al., Cationic liposomes coated with polyethylene glycol as carriers for oligonucleotides, J. Biol. Chem. 273 (25) (1998) 15621-15627.

[46] S. Mishra, P. Webster, M.E. Davis, PEGylation significantly affects cellular uptake and intracellular trafficking of non-viral gene delivery particles, Eur. J. Cell Biol. 83 (3) (2004) 97-111.

[47] F.X. Shi, L. Wasungu, A. Nomden, et al., Interference of poly(ethylene glycol)-lipid analogues with cationic-lipid-mediated delivery of oligonucleotides; role of lipid exchangeability and non-lamellar transitions, Biochem. J. 366 (2002) 333-341.

[48] L.Y. Song, Q.F. Ahkong, Q. Rong, et al., Characterization of the inhibitory effect of PEG-lipid conjugates on the intracellular delivery of plasmid and antisense DNA mediated by cationic lipid liposomes, Biochim. Biophys. Acta Biomembr. 1558 (1) (2002) 1-13.

[49] K. Buyens, B. Lucas, K. Raemdonck, et al., A fast and sensitive method for measuring the integrity of siRNA-carrier complexes in full human serum., J. Control. Release (in press), doi:10.1016/j.jconrel.2007.10.024.

[50] C.T. Moonen, Spatio-temporal control of gene expression and cancer treatment using magnetic resonance imaging-guided focused ultrasound, Clin. Cancer Res. 13 (12) (2007) 3482-3489. 